\title{
Antiphospholipid antibody associated thrombosis in juvenile chronic arthritis
}

\author{
Roberto Caporali, Angelo Ravelli, Barbara Ramenghi, Carlomaurizio Montecucco, \\ Alberto Martini
}

\begin{abstract}
A child with systemic onset juvenile chronic arthritis (JCA) who developed a bilateral femuropopliteal vein thrombosis after plaster immobilisation following a tibial fracture is described. When the thrombosis was diagnosed, antiphospholipid antibodies detected either as lupus anticoagulant and anticardiolipin antibodies were found. This suggests that short term prophylactic antithrombotic treatment should be considered in antiphospholipid antibody positive JCA patients who require immobilisation after fractures of demineralised bones.
\end{abstract}

\section{(Arch Dis Child 1992;67:1384-6)}

There is evidence that patients with antiphopholipid antibodies, detected as lupus anticoagulant (LAC) or as anticardiolipin antibodies $(\mathrm{aCL})$, have a tendency to thromboembolic phenomena, both arterial and venous. However, follow up studies have shown that only a minority of patients with these antibodies develop thrombosis, ${ }^{1}$ and the real predictive value of antiphospholipid antibodies for developing thrombosis is not yet established.

Recently, we found a high prevalence of aCL in patients with juvenile chronic arthritis (JCA). ${ }^{2}$ However, we failed to demonstrate any relationship between aCL and those clinical manifestation usually associated with these antibodies. Moreover, to the best of our knowledge, no case of antiphospholipid antibody associated thrombosis reported in children had JCA. ${ }^{3}$

We describe here a child with systemic onset JCA who developed a bilateral femuropopliteal vein thrombosis after plaster immobilisation following a tibial fracture. At the time when the thrombosis was diagnosed, we found the presence of both LAC and aCL. Our case suggests that antiphospholipid antibodies may be an important cofactor for developing thrombosis in children with JCA when another potentially thrombophilic event occurs.

Italy,

Institute of

Medical Pathology

Roberto Caporali

Carlomaurizio Montecucco

Paediatric Clinic

Angelo Ravelli

Barbara Ramenghi

Alberto Martini

Correspondence to:

Professor A Martini,

Clinica Pediatrica,

Università di Pavia,

IRCCS S Matteo,

Ple Golgi 2

27100 Pavia,

Italy.

Accepted 1 July 1992 years the disease remained active with persistent systemic manifestations and severe polyarthritis. In the meantime, the boy progressively
In 1978 a boy, aged 4 years, presented with high sis of systemic onset JCA was made in our with salicylates, auranofin and, during systemic and/or articular exacerbations, with cycles of

\section{Case report}

developed multiple limitations of joint motion and diffuse radiological signs of articular damage. During this time he was seldom referred to our department and the dosage and regimen of low dose prednisone were managed by his parents at home independently, according to his daily symptoms. In September 1988, 10 years after onset, he had a cushingoid appearance, with multiple limitations of joint motion and severe difficulty in walking, and persistence of fever, rash, and polyarticular synovitis. Skeletal radiograms revealed a dorsolumbar vertebral collapse, worsening of articular erosions, particularly at the hips, and diffuse osteoporosis. Steroids were suspended and low dose oral methotrexate $\left(10 \mathrm{mg} / \mathrm{m}^{2} /\right.$ week $)$ was started in association with salicylates.

In May 1990 the child was involved in a road accident and suffered a multiple fracture of the right tibia. He was treated elsewhere with exceedingly prolonged (five months) plaster immobilisation of the right leg. After plaster removal a generalised swelling of the right leg with cutaneous erythema and local pain was noted. The child came back to our department three months later. Physical examination showed swelling of both legs, with the circumference of the right $3 \mathrm{~cm}$ greater than the contralateral, slight cutaneous redness and, on the right side, a subcutaneous venous reticulum and oedema of the dorsum of the foot. Examination revealed multiple limitations of joint motion and active synovitis at wrists, knees, and ankles.

Pertinent laboratory investigations were as follows: white cell count $13 \times 10^{9} / 1$ with normal differential, haemoglobin concentration $109 \mathrm{~g} / \mathrm{l}$, platelet count $833 \times 10^{9} / 1$, erythrocyte sedimentation rate $60 \mathrm{~mm}$ in the first hour, prothrombin time $50 \%$ (normal range $70-110 \%$ ), partial thromboplastin time 75.4 seconds (normal $<26$ seconds). Blood urea, creatinine, glucose, electrolytes, and serum complement

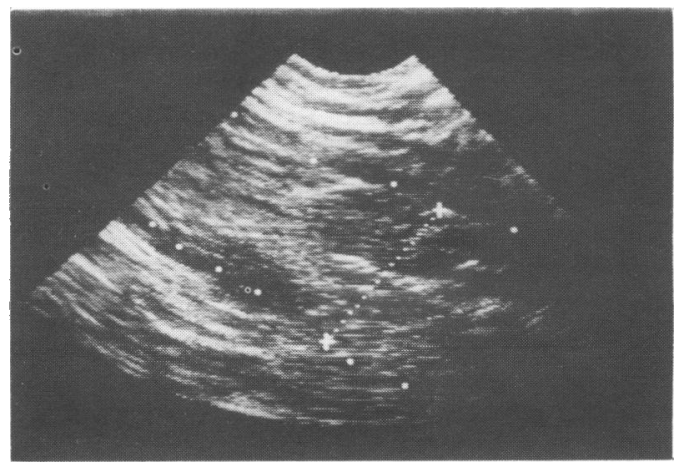

Doppler ultrasonography of the left leg showing a thrombotic occlusion of the popliteal vein. 
concentrations, and urinalysis were normal. Rheumatoid factor and antinuclear, antiextractable nuclear antigen, and antimitochondrial antibodies were absent. The LAC test was positive and low titre ( 12 arbitrary units) of aCL of the IgG isotype were found by enzyme linked immunoabsorbent assay. Both LAC and aCL tests were performed as previously described. ${ }^{4}$ A Doppler ultrasound examination of the lower limbs showed an extensive, bilateral thrombosis of both femoral and popliteal veins (figure). The child was discharged with weekly subcutaneous heparin calcium (Calciparine, Sanofi) and was then lost to follow up.

\section{Discussion}

We have reported a child with systemic onset JCA who developed an extensive deep vein thrombosis after a five month period of plaster immobilisation following a tibial fracture. When thrombosis was diagnosed, three months after plaster removal, both LAC and low titre IgG aCL were detected.

Only a few data exist on antiphospholipid antibodies in childhood. Our recent studies have shown a high frequency of aCL in children with $\mathrm{JCA}^{2}$ but no $\mathrm{aCL}$ associated thrombotic events have been observed in these cases. Therefore, in JCA, the real predictive value of aCL antibodies for developing thromboembolic events is weak, and in no way is antithrombotic prophylaxis indicated on the unique basis of a positive aCL assay.

It should be noted, however, that the clinical experience on aCL in adults has shown that associated thrombophilic factors such as smoking and use of oral contraceptives may enhance the risk of thrombosis. ${ }^{5}$ Prolonged immobilisation is well known to be associated with an increased risk of thromboembolism, and its association with antiphospholipid antibodies might have lead to deep vein thrombosis in our child.

This observation suggests that when children with JCA need immobilisation, such as after fractures of demineralised bones, the presence of antiphospholipid antibodies should be investigated as they may act as additional risk factors for thrombosis.

It should be noted that in our patient both LAC and $\mathrm{aCL}$ were detected and that it has been suggested that LAC represents a more specific, although less sensitive, marker of the thrombotic risk than aCL. ${ }^{6}$

In conclusion, our report suggests that short term prophylactic anticoagulant and/or antiaggregant treatment should be considered in those antiphospholipid antibody positive children with JCA who require immobilisation, particularly if circulating LAC is present.

1 Love PE, Santoro SA. Antiphospholipid antibodies, anticardiolipin and the lupus anticoagulant in systemic lupus erythematosus (SLE) and in non-SLE disorders. Prevalence and clinical significance. Ann Intem Med 1990,112.682-98.

2 Caporali R, Ravelli A, De Gennaro F, Neirotti G, Montecucco C, Martini A. Prevalence of anticardiolipin antibodies in juvenile chronic arthritis. Ann Rheum Dis 1991;50:599-601.

3 Montes de Oca M, Babron MC, Bletry $\mathrm{O}$, et al. Thrombosis in systemic lupus erythematosus: a French collaborative study. Arch Dis Child 1991;66:713-7.

4 Ravelli A, Caporali R, Bianchi E, et al. Anticardiolipin syndrome in childhood: a report of two cases. Clin Exp Rheumatol 1990;8:95-8.

5 Asherson RA, Harris EN, Hughes GRV. Complication of oral contraceptives and antiphospholipid antibodies. Arthrtis Rheum 1988;31:575-6.

6 Mackworth-Young C. Antiphospholipid antibodies: more than just a disease marker? Immunol Today 1990;11:605.

\title{
Severe anaemia and ileocolic anastomotic ulceration
}

\author{
A H Hamilton, J M Beck, G M Wilson, H J Heggarty, J W L Puntis
}

\section{Abstract}

Two children are described with anaemia from ileocolic anastomosic ulceration as a late complication of surgery in the newborn period. The anastomosis was revised in each case but in one child there was early recurrence of ulceration.

\section{(Arch Dis Child 1992;67:1385-6)}

Ulceration at the site of an ileocolic anastomosis as a late complication of neonatal surgery has been described, but is probably underrecognised. Rational management is hampered by lack of understanding of the underlying aetiology of the condition. We report our experience in two children with this unusual problem.

\section{Case reports}

CASE 1

A boy of 25 weeks' gestation required ventilation for respiratory distress, but after extubation he was established on full enteral feeds. At 9 weeks of age he developed abdominal distension and bloody diarrhoea and an abdominal $x$ ray film confirmed necrotising enterocolitis. Despite medical management his condition deteriorated, necessitating laparotomy. A diseased segment of ileum was resected and a cutaneous enterostomy fashioned, after which he made a good recovery. Two months later radiological investigation confirmed two colonic strictures that were resected and an ileocolic anastomosis performed. He was discharged home at 6 months of age, on full enteral feeding, and followed up at his local hospital.

He was referred to the surgical unit at the age of 7 years for investigation of anaemia. He had no symptoms, was on the third centile for both height and weight, and was passing two normal stools each day. Despite recent transfusion, investigation revealed a microcytic hypochromic anaemia, with a haemoglobin concentration of $65 \mathrm{~g} / \mathrm{l}$. Testing for faecal occult blood gave a strongly positive result. An upper gastrointestinal endoscopy and barium meal both gave normal results, but at colonoscopy the mucosa 